\title{
Drug overdose due to malfunction of a patient-controlled analgesia machine -A case report-
}

\author{
Yuri Yi, Seongsik Kang, and Byeongmun Hwang \\ Department of Anesthesiology and Pain Medicine, Institute of Medical Sciences, Kangwon National University Hospital, School of \\ Medicine, Kangwon National University, Chuncheon, Korea
}

Patient-controlled analgesia (PCA) provides excellent pain control and high stability, thereby minimizing the incidence of adverse effects. However, one of our patients experienced respiratory depression and hypotension within 30 minutes of initiation of PCA therapy. We discovered that machine malfunction caused continuous activation of the PCA button, resulting in a drug overdose. The PCA machine was sent to the manufacturer, who found an electrical short in the PCA button. All PCA units of the same make and model were immediately removed from hospitals and replaced with redesigned units without defects in the PCA button. We have used the improved machines without any problems. The purpose of this report is to raise awareness of this type of malfunction in PCA units in order to help prevent adverse events in the future. (Korean J Anesthesiol 2013; 64: 272-275)

Key Words: Electrical short circuit, Hypotension, Opioid, PCA, Respiratory depression.

Patient-controlled analgesia (PCA) is a delivery system with which patients self-administer predetermined doses of analgesic medication for pain relief. Compared to conventional pain treatment, PCA improves analgesia and decreases the risk of pulmonary complications [1]. However, several cases of PCArelated adverse effects have been reported [2-4]. Recently, a patient in our hospital experienced respiratory depression and hypotension associated with PCA. Herein, we describe the case, discuss its causes, and propose helpful guidelines for use of PCA machines.

\section{Case Report}

A 71-year-old woman (height, $154 \mathrm{~cm}$; weight, $57 \mathrm{~kg}$ ) underwent vaginal total hysterectomy and anterior-posterior vaginal wall repair for uterine prolapse. Her medical history was unremarkable. Findings of routine preoperative laboratory tests, chest radiography, and electrocardiography were within normal limits.

The patient received general anesthesia for the procedure. The duration of anesthesia was 150 minutes. Estimated blood

Received: February 29, 2012. Revised: April 20, 2012. Accepted: May 23, 2012.

Corresponding author: Byeongmun Hwang, M.D., Ph.D., Department of Anesthesiology and Pain Medicine, Kangwon National University Hospital, School of Medicine, Kangwon National University, Chuncheon 200-701, Korea. Tel: 82-33-258-2238, Fax: 82-33-258-2271, E-mail: arim14@kangwon.ac.kr

(c) This is an open-access article distributed under the terms of the Creative Commons Attribution Non-Commercial License (http:// creativecommons.org/licenses/by-nc/3.0/), which permits unrestricted non-commercial use, distribution, and reproduction in any medium, provided the original work is properly cited. 
loss was $200 \mathrm{ml}$, and the infused fluid was Hartmann solution $(1,500 \mathrm{ml})$. There were no adverse events or complications during anesthesia and surgery. The patient was transferred to the post-anesthesia care unit. An intravenous PCA machine (AutoMed3400 ${ }^{\circledR}$ Ace Medical, Seoul, Korea) was connected, and the nurse pushed the button once. The PCA solution contained 1,000 $\mu$ g fentanyl (Fentanyl citrate ${ }^{\circledR}$ HanLim Pharm, Korea), 120 mg tarasyn (Ketorolac Tromethamine ${ }^{\circledR}$ Roche Pharm, Korea), and $32 \mathrm{mg}$ ondant (Ondansetron ${ }^{\circledR}$ Hanmi Pharm, Korea) mixed with $100 \mathrm{ml}$ normal saline in a total volume of $140 \mathrm{ml}$. The bolus dose was $2 \mathrm{ml}$. The PCA was set at a basal rate of $2 \mathrm{ml} / \mathrm{hr}$, with a lock-out time of 12 minutes. The patient complained of being cold, so an air warmer was applied. No difficulties were observed. The patient's mental functions were intact, and her vital signs were stable. Blood pressure (BP) was 120/75 mmHg; heart rate (HR), $65 \mathrm{bpm}$; and oxygen saturation $\left(\mathrm{SpO}_{2}\right), 99 \%$.

After 30 minutes, the nurse was alerted by an alarm from the pulse oximeter indicating an $\mathrm{SpO}_{2}$ of $80 \%$. The patient was unresponsive and had a very low respiratory rate. Her BP had decreased to $65 / 48 \mathrm{mmHg}$. Immediate resuscitative measures were performed. Ephedrine (30 mg) was administered intravenously. The patient was intubated and manually resuscitated for 10 minutes. The PCA machine indicator showed a total infused volume of $31 \mathrm{ml}$, indicating that the patient had received $221 \mu \mathrm{g}$ fentanyl and $26 \mathrm{mg}$ tarasyn. The patient was immediately disconnected from the PCA machine. Fortunately, the patient showed rapid improvement in consciousness and ventilation. In 20 minutes, she was fully alert, and her vital signs had stabilized. Her BP was 130/80 mmHg; HR, 80 bpm; and $\mathrm{SpO}_{2}, 100 \%$. Extubation was performed, and close observation was maintained. The patient was sent to the ward and discharged from the hospital 1 week later, without further complications.
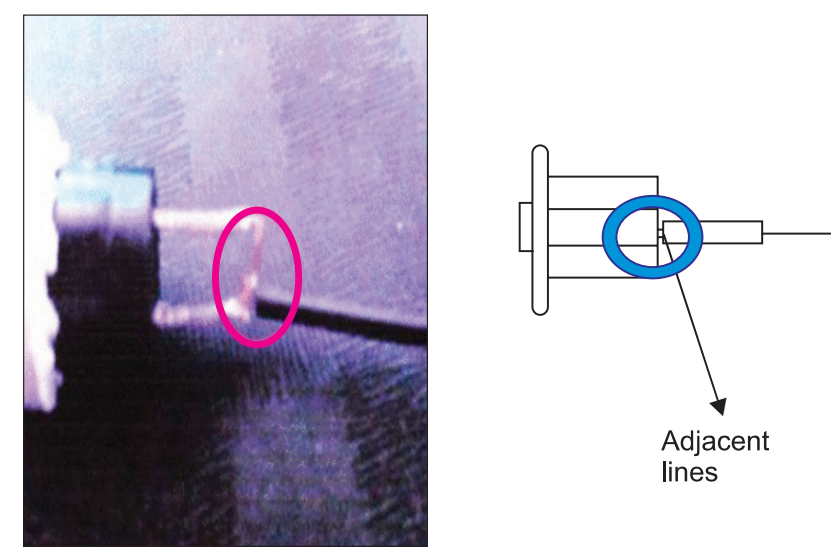

Fig. 1. Cable in the press button is misplaced, allowing wires from both sides to touch one another. Circled area depicts the area in which the electrical short circuit occurred.

\section{Discussion}

Since its introduction 25 years ago, PCA has become the gold standard for acute pain management. Its merits have been discussed in many publications $[1,5,6]$. The principle of PCA is to allow the patient to choose when to administer a bolus of analgesic. Self-administration provides a sense of well-being to patients because they are able to control their pain unassisted. The inherent safety mechanism in PCA is that as patients selfadminister small doses of opioids, they become progressively sedated. Patients thus cease demand before their blood opioid concentrations increase to toxic levels [7].

In general, the efficiency and safety of PCA in the treatment of postoperative pain is well-documented [6]. However, some studies have reported safety hazards and deaths from fatal opioid overdose associated with PCA machines [8]. Opioid administration is associated with alterations in ventilation. Lifethreatening respiratory events associated with the use of PCA have been reported [9]. These events are most often the result of human error, usually related to machine programming.

In the current case, over-infusion of drug was due to machine malfunction. Immediately after connecting the PCA machine to the patient, the activation button was pushed just once. Had the machine functioned correctly, the total infused volume after 30 minutes would have been $3 \mathrm{ml}$, containing a total of $21 \mu \mathrm{g}$ fentanyl and $2.5 \mathrm{mg}$ tarasyn. When we found the patient unresponsive, the patient's PCA machine indicated a total infused volume of $31 \mathrm{ml}$, implying that the drug had been administered continuously after the first activation. The cause of this malfunction was an electrical short circuit in the PCA machine.

This PCA machine had been used for several years in our hospital without the occurrence of such an episode. We immediately contacted the PCA manufacturer, and they collected the machine. The manufacturer's medical engineering
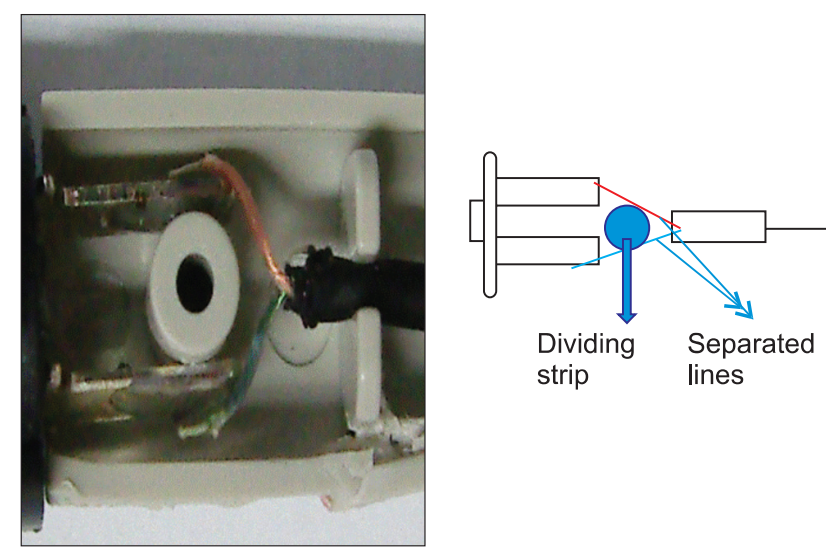

Fig. 2. Double-pole, single-throw push button with 2 signal lines. 
team reported that a cable in the activation button was out of place, causing an electrical short circuit (Fig. 1). This resulted in continuous activation of the machine and over-infusion of drug. All potentially problematic PCA machines were removed from use. Several months later, the PCA manufacturer announced that the defective button had been replaced with a double-pole, single-throw push button and 2 signal lines in order to prevent electrical shorts (Fig. 2). To date, the company reports that the upgraded PCA machines have not experienced electrical shorts in the activation mechanism.

A short circuit is an abnormal low-resistance connection between 2 nodes of an electrical circuit intended to be at different voltages. Unintentional short circuits usually occur when the wire's insulation breaks down, or when another conduction material is introduced. This results in an excessive electric current that flows along an unintended path; this current can cause circuit damage, overheating, fire, or explosion. In the present case, a cable in the PCA activation button was out of place for unknown reasons, causing a short circuit. The picture sent by the PCA manufacturer showed the wires that were fused because of fire or explosion (Fig. 1).

Although we encountered an unexpected and hazardous event due to PCA machine malfunction, this situation provided a positive long-term result. The PCA manufacturer removed defective machines from the market and introduced upgraded PCA machines. The defective button was replaced with a double-pole, single-throw push button and 2 signal lines in order to prevent an electrical short. The re-designed machine is free from the short-circuiting problems inherent in the earlier model.

Electronic PCA devices allow flexibility in the timing and amounts of delivered doses. They can also be programmed to deliver constant background infusions. They are designed to be tamper-proof, so that access to the drug without a key is theoretically impossible without damaging the pump in the attempt [10]. However, successful keyless access has been achieved by unauthorized personnel without machine breakage. Additionally, reports of problems related to the use of electronic PCA devices are common.

Elastomeric pump-controlled PCA devices are alternative pain management tools. Elastomeric pumps have several advantages over electronic pumps, including portability, ease of use, and fewer technical problems such as undesirable alarm triggering [11]. Furthermore, the device delivers a fixed volume with each demand, and the amount of drug delivered in each bolus can only be altered by changing the concentration of drug in the reservoir. In addition, the opioid in the reservoir is much more easily accessible than that in locked electronic infusion devices. Elastomeric pumps are maintenance-free and run independently of electronics. These pumps are typically single- use and disposable. We propose that this type of pump is ideal for outpatients or patients requiring high levels of mobility.

The pressure required for administering the drug comes from the elastomeric layer that is molded inside the pump. When the pump is filled, the layer is stretched. The elastic constriction drives the liquid through the tubing and eventually through a flow restrictor out into the patient connection. The pressure is consistent until near the end of the infusion, when there is usually a pressure spike resulting in a higher flow rate. The accuracy of the flow rate is controlled by a flow restrictor that is molded into the tubing of the system or placed within the elastomeric reservoir.

However, elastomeric PCA devices have several disadvantages [12]. One is the absence of a lock-out function. Without a lockout function, it is possible to administer a small amount of drug by pushing the PCA button very frequently. In such a case, the effect of bolus injection contributing to a wider distribution of the administered drug might be sacrificed. In addition, the absence of a recording function can be a problem. It is almost impossible to know the number of PCA attempts made by patients. The only recording method is to routinely weigh the PCA pump to detect infusion abnormalities. The absence of an alarm is another concern. Malfunctions resulting in overdosing or occlusion are difficult to detect immediately.

Considering all of the aspects of this case, we propose the following changes in PCA use. The first is incorporation of a mechanical safety device that is resistant to electrical disturbances in order to prevent continuous over-infusion [13]. Indeed, this is what the PCA manufacturer did. Second, we propose incorporation of a function that alerts providers when programmed doses exceed safe limits. Regardless of the basal rate, bolus dose, and lock-out time, the dose of drug administered over a 1-hour period should be limited. This acts as a back-up safety check to prevent drug overdose, regardless of whether the machine is malfunctioning or the patient is activating the system excessively. Use of elastomeric PCA device might be a solution to this problem. However, there are some flaws in elastomeric PCA device, as previously discussed. Finally, we recommend that medical staff respond rapidly to device alarms. We understand that this approach does not solve the problem, but prompt action is essential when things are going wrong. In the present case, less than 3 minutes passed after the pulse oximeter alarm was heard before the patient was intubated. We are confident that the prompt reaction was responsible for the patient's rapid improvement and discharge, without further complications, in 1 week.

We believe that PCA devices have greater safety in continuously monitored hospital settings during the entire course of a patient's use. Risks related to PCA device failure might also support the use of ward-based continuous oximetry 
devices [14].

We reported a case of respiratory depression and hypotension caused by continuous over-infusion of opioid due to PCA machine malfunction. Immediate resuscitative measures ensured that no sequelae occurred. However, when using PCA, practitioners should have working knowledge of the PCA machine, including machine types, advantages and disadvantages of each type, and the possible complications associated with each type. Although PCA is well known for safety, intense monitoring of patients is necessary in case of unexpected situations. With focused efforts to understand PCA machines and continued attention to patients, PCA can be an excellent method for effective and safe control of postoperative pain.

\section{References}

1. Walder B, Schafer M, Henzi I, Tramèr MR. Efficacy and safety of patient-controlled opioid analgesia for acute postoperative pain. A quantitative systematic review. Acta Anaesthesiol Scand 2001; 45: 795-804.

2. Schein JR, Hicks RW, Nelson WW, Sikirica V, Doyle DJ. Patient controlled analgesia related medication errors in the postoperative period: causes and prevention. Drug Saf 2009; 32: 549-59.

3. Musshoff F, Padosch SA, Madea B. Death during patient-controlled analgesia; piritramide overdose and tissue distribution of the drug. Forensic Sci Int 2005; 154: 247-51.

4. Chung HS, Kim ES, You YJ, Park CS. Anaphylactoid reaction after injection of ketorolac in a loading dose for patient-controlled analgesia. Korean J Anesthesiol 2010; 58: 565-8.

5. Lehmann KA. Recent developments in patient-controlled analgesia. J Pain Symptom Manage 2005; 29 (5 Suppl): S72-89.

6. Etches RC. Patient-controlled analgesia. Surg Clin North Am 1999; 79: 297-312.

7. Mercadante S. Intravenous patient-controlled analgesia and management of pain in post-surgical elderly with cancer. Surg Oncol 2010; 19: 173-7.

8. Vicente KJ, Kada-Bekhaled K, Hille G, Cassano A, Orser BA. Programming errors contribute to death from patient-controlled analgesia: case report and estimate of probability. Can J Anaesth 2003; 50: 328-32.

9. Bailey P. Opioid-induced respiratory depression. Prog Anesthesiol 1991; 5: 170-84.

10. Macintyre PE. Safety and efficacy of patient-controlled analgesia. Br J Anaesth 2001; 87: 36-46.

11. Zahnd D, Aebi S, Rusterholz S, Fey MF, Borner MM. A randomized crossover trial assessing patient preference for two different types of portable infusion-pump devices. Ann Oncol 1999; 10: 727-9.

12. Sumikura H, van de Velde M, Tateda T. Comparison between a disposable and an electronic PCA device for labor epidural analgesia. J Anesth 2004; 18: 262-6.

13. Notcutt WG, Knowles P, Kaldas R. Overdose of opioid from patientcontrolled analgesia pumps. Br J Anaesth 1992; 69: 95-7.

14. Overdyk FJ, Carter R, Maddox RR, Callura J, Herrin AE, Henriquez C. Continuous oximetry/capnometry monitoring reveals frequent desaturation and bradypnea during patient-controlled analgesia. Anesth Analg 2007; 105: 412-8. 